\title{
Traduire
}

Revue française de la traduction

$215 \mid 2007$

La qualité en perspective

\section{La veille sanitaire et médicale : bref aperçu}

\section{Sophie Terquem}

\section{(2) OpenEdition}

\section{Journals}

Édition électronique

URL : http://journals.openedition.org/traduire/1351

DOI : 10.4000/traduire. 1351

ISSN : 2272-9992

\section{Éditeur}

Société française des traducteurs

\section{Édition imprimée}

Date de publication : 1 décembre 2007

Pagination : 76-88

ISSN : 0395-773X

\section{Référence électronique}

Sophie Terquem, «La veille sanitaire et médicale : bref aperçu », Traduire [En ligne], 215 | 2007, mis en ligne le 01 décembre 2007, consulté le 19 avril 2019. URL : http://journals.openedition.org/ traduire/1351 ; DOI : 10.4000/traduire.1351 


\title{
La veille sanitaire et médicale : bref aperçu
}

\author{
SOPHIE TERQUEM \\ Établissement public de santé Paul Guiraud
}

S'il existe un domaine dans lequel la veille revêt une importance particulière, c'est bien le domaine de la santé et de la médecine. Les professionnels de santé et les médecins en sont conscients depuis fort longtemps mais les décideurs publics et privés tardent à mettre en place une véritable stratégie de veille, commune et coordonnée, pour diverses raisons qu'il serait long de développer ici. Signalons simplement que le caractère novateur de la "fonction veille " explique en partie cette absence de coordination, d'autant plus que l'activité est fort peu connue du grand public bien qu'elle soit très utile dans la pratique.

L'objectif premier de cet article est de présenter de façon aussi concrète que possible les institutions et les outils existants pour la veille sanitaire et médicale, l'idée étant de montrer leur caractère opérationnel mais aussi l'urgence d'une politique volontariste en la matière. Cette vision politique se justifie d'autant plus que la veille sanitaire et médicale se situe au croisement de plusieurs domaines stratégiques qui forment le cœur des préoccupations sociales de nos jours : la santé publique et les nouvelles technologies.

\section{Une activité indispensable}

En matière de médecine et de santé publique, tout le monde s'accorde sur le fait que le volume des informations disponibles est déjà considérable et ne cesse de croittre à un rythme exponentiel. Dans ce contexte d'explosion informationnelle, espérer maîtriser avec les moyens humains existants ce flux continu de données parfois critiques et 
souvent indispensables à la gestion des problèmes de santé publique, relève de l'utopie. Pour y parvenir, il est évident qu'il faudrait une stratégie de veille appuyée sur des outils informatiques et des logiciels performants.

Le souvenir des crises survenues, dans un passé pas si lointain, doit d'ailleurs nous inciter à la vigilance et au perfectionnement du système actuel. De la gestion " artisanale " de la canicule de 2003 jusqu'aux menaces de pandémie grippale, en passant par les campagnes de santé publique sur le tabagisme ou l'alcoolisme, tout démontre que nous avons de plus en plus besoin d'un véritable système de prospective et d'une nouvelle stratégie de veille en matière de santé publique.

Cette conscience des limites de notre fonctionnement actuel est née de deux expériences : la première réside dans la découverte de ce qui se fait ailleurs que chez nous en matière de veille sanitaire et médicale, en particulier outre-atlantique aux États-Unis et au Canada ; la seconde concerne la découverte des activités de veille systématique et multilingue qui sont pratiquées dans d'autres domaines que la santé et qui démontrent un dynamisme encourageant et une utilité indéniable de la fonction veille.

Mais répondons d'abord à des questions simples : qu'est-ce que la veille sanitaire et quelle est la situation actuelle ?

La veille sanitaire est l'action de surveiller l'état de santé d'une population dans un but de prévention (épidémies ou tout autre problème de santé publique).

La veille médicale est plus spécifique puisqu'elle porte sur le suivi régulier des publications et des innovations en matière de médecine.

Les équivalents anglais (Health Watch, Medical Watch) indiquent bien l'étendue du domaine sur lequel porte la veille sanitaire et médicale.

Ces deux types de veilles interconnectées ne peuvent plus se faire aujourd'hui avec de simples moyens humains mais nécessitent le recours à des outils logiciels de plus en plus complexes. 
Cela est d'autant plus vrai que les activités de veille sanitaire sont aujourd'hui réparties entre plusieurs institutions dont nous donnons ci-après un bref aperçu.

\section{Un paysage de veille éclaté}

Sommairement, disons que la veille est répartie aujourd'hui entre un institut national (InVS) et trois agences principales (AFSSA, AFSSAPS, AFSSET), tous issus de la loi du 1er juillet 1998 relative à la veille sanitaire, à la suite de la crise de la vache folle.

L'Institut de veille sanitaire (InVS) est un établissement public directement rattaché au ministère de la Santé. Sa mission première est de surveiller l'état de santé de la population et d'alerter les pouvoirs publics, les professionnels de la santé et l'ensemble de la population, en cas de nécessité, c'est-à-dire de menace d'épidémie par exemple.

Les agences françaises de sécurité sanitaire sont également des établissements publics dont la mission principale est d'évaluer les risques sanitaires.

1) L'Agence française de sécurité sanitaire des aliments (AFSSA)(1) évalue les risques sanitaires et nutritionnels présentés par tous les aliments, y compris l'eau, et cela dans le but d'alerter les pouvoirs publics et, plus largement, d'informer la population. Ainsi, c'est l'AFSSA qui a recommandé l'arrêt d'importation en France de viande bovine aux hormones en provenance des États-Unis et de GrandeBretagne pour cause d'encéphalopathie spongiforme bovine (ESB). Mais cette recommandation est le résultat d'une veille intensive et d'un suivi minutieux de la situation dans les deux pays concernés par l'interdiction.

Pour évaluer les risques nutritionnels et sanitaires, la veille doit en effet intégrer l'ensemble de la chaîne alimentaire et s'exercer sur chacune de ses étapes, de la production à la consommation. La décision de recommander l'interdiction est le fruit de ce suivi.

(1) Voir le site de l'agence à l'adresse suivante : http://www.afssa.fr/ 
La veille sanitaire intègre ainsi une mission de recherche documentaire et d'appui scientifique notamment en matière de santé animale et de maladies d'origine animale, les médicaments vétérinaires, les pesticides, les produits phytopharmaceutiques, ou encore les produits phytosanitaires.

En raison de ces champs de compétences, la veille exercée dans le cadre de l'AFSSA est menée par des comités spécialisés relevant de dix domaines principaux : nutrition humaine, alimentation animale, microbiologie, biotechnologie et organismes génétiquement modifiés (OGM), santé animale, eaux, résidus et contaminants chimiques et physiques ; additifs, arômes et auxiliaires technologiques, matériaux au contact des denrées alimentaires.

En ce qui concerne la veille multilingue exigée par le domaine même de la santé publique dont les risques ne s'arrêtent pas aux frontières nationales, l'AFSSA dispose d'un réseau de correspondants à l'étranger. Ceux-ci relèvent essentiellement d'autorités institutionnelles européennes (telle que l'Autorité européenne de sécurité des aliments) ou gouvernementales (telle que la Food and Drugs Administration aux États-Unis).

2) L'Agence française de sécurité sanitaire des produits de santé (AFSSAPS)(2) a pour mission principale d'évaluer les risques sanitaires présentés par les médicaments. Mais elle mène également des activités de veille concernant les matières premières, les dispositifs médicaux, les dispositifs de diagnostic in vitro, les produits biologiques d'origine humaine (produits sanguins labiles, organes, tissus, cellules, produits de thérapie génique et de thérapie cellulaire), les produits thérapeutiques annexes, ou encore les produits cosmétiques.

Cette veille est essentielle parce qu'elle vise, entre autres, l'évaluation scientifique et médico-économique, le contrôle de la publicité dans le domaine médical, les recommandations sur le bon usage des médicaments, le contrôle de la publicité en faveur des

(2) Voir le site de l'agence à l'adresse suivante : http://afssaps.sante.fr/ 
objets, appareils et méthodes présentés comme bénéfiques pour la santé, l'information des professionnels de santé et du public sur les avantages et les risques.

Pour mener ses activités de veille, l'AFSSAPS s'appuie sur des commissions spécialisées dans de nombreux domaines dont les suivants : la pharmaco-vigilance, la matério-vigilance, la cosmétologie, la pharmacopée, les stupéfiants et les psychotropes, les réactifs, etc. Comme l'agence de sécurité des aliments et pour les mêmes raisons de perméabilité des frontières aux risques sanitaires, l'AFSSAPS possède des correspondants à l'étranger, en particulier aux ÉtatsUnis (Food and Drugs Administration, FDA) et au Royaume-Uni (Food Standards Agency, FSA). Malgré cela, la dimension multilingue de ses activités de veille sanitaire et médicale demeure très modeste.

3) L'Agence française de sécurité sanitaire de l'environnement et du travail (AFSSET)(3) contribue à assurer la sécurité sanitaire dans le domaine de l'environnement. Ses activités de veille visent à évaluer les risques sanitaires liés à l'environnement et à fournir au gouvernement l'expertise et l'appui scientifique et technique nécessaires à l'élaboration et à la mise en œuvre des dispositions législatives et réglementaires relevant de la santé de l'environnement et du travail. L'AFSSET coordonne l'expertise d'évaluation des risques liés aux produits chimiques, avec une vingtaine d'organismes scientifiques tels que l'INRS ou l'INSERM. Elle rassemble les rapports d'expertise en santé de l'environnement et en santé au travail, mais elle a aussi une mission d'information sur ces thèmes.

La partie multilingue de son activité est, à ce jour, rudimentaire, mais elle est appelée à se développer avec l'ouverture et l'élargissement du marché de travail européen.

Toutes ces agences sont aujourd'hui au cœur d'un réseau d'information qui reste largement unilingue et national. Or, la veille 
multilingue est une ressource pour l'action qui permet de faire mieux, plus vite et de façon plus innovante. Elle amplifie les capacités d'information et, du coup, la maîtrise des attributs informatiques des données (texte, son, image).

Cette dimension de la veille - multilingue et multimédia - est largement négligée parce que la question de ses usages professionnels reste à construire à travers une collaboration étroite entre professionnels de la santé et experts de la veille. Pour tirer le meilleur parti des puissants outils de recherche d'information existants, il faut développer une compétence technologique avancée qui intègre au moins la mise à profit des nombreuses bases de données et services relatifs à la veille sanitaire et médicale.

En nous appuyant sur une investigation menée au cours de l'automne 2007 , nous proposons ci-après un aperçu des potentialités de veille liées à Internet. Les outils présentés ci-après visent à montrer les modalités pratiques des usages professionnels de la veille et à illustrer en quoi la veille multilingue constitue un saut qualitatif important en matière de veille sanitaire et médicale.

\section{Des outils de veille sous exploités}

Ce qui différencie les activités de veille de la recherche documentaire classique, c'est qu'elles s'inscrivent dans la durée et qu'elles intègrent une dimension stratégique forte. En effet, si l'action de se documenter sur un sujet s'exerce de façon ponctuelle (événement majeur, crise de santé, catastrophe naturelle, épidémie...), l'activité de veille consiste, au contraire, au maintien de la recherche d'informations dans le temps, en employant des méthodes et des outils automatisés.

La veille "classique " est bien connue dans le domaine sanitaire et médical, mais les méthodes et outils disponibles pour pratiquer la veille automatique le sont beaucoup moins parce qu'ils sont relativement récents et peu usités, malgré leur intérêt indéniable.

La veille automatique permet notamment de suivre dans le temps les évolutions d'une technologie particulière ou les nouveautés techniques 
en matière de santé publique, de surveiller le contenu des publications spécialisées sur le domaine, de créer une revue de presse ciblée ou encore de mettre à jour régulièrement un site Web institutionnel.

Il est clair que cette veille informationnelle peut être réalisée avec des moyens humains (documentalistes, experts, consultants), mais il est également évident que le recours aux techniques de surveillance automatisée peut faciliter et enrichir grandement la tâche. Cela est d'autant plus vrai que les veilleurs ont à leur disposition une panoplie d'outils de plus en plus performants. Leur intérêt réside dans le fait qu'ils permettent d'accomplir plusieurs types d'actions qui s'avèrent fastidieuses pour l'agent humain :

1) La répétition d'une même recherche à l'infini : une action de veille automatique peut s'exécuter par la répétition d'une même recherche sur un ou plusieurs outils. Cela peut consister à répéter une recherche donnée à intervalles réguliers ou bien à interroger de façon systématique les mêmes sources d'informations.

2) Le suivi des pages Web : la surveillance des modifications et des nouveautés qui apparaissent sur certains sites choisis est facilitée par le recours à des outils dédiés à cette tâche, tels que les aspirateurs de sites (comme MemoWeb ou TeleportPro) et les agents de veille (comme WebSiteWatcher). Les aspirateurs permettent de rapatrier tout ou partie d'un site Web sur l'ordinateur, à intervalles réguliers, afin d'en analyser les modifications. Les agents de veille permettent de " pister " l'apparition de mots clés spécifiques sur les pages surveillées.

3) Le Webcasting consiste à s'abonner à des canaux d'information sur une ou plusieurs thématiques données et à recevoir automatiquement les dernières nouvelles sur les canaux en question. Les domaines couverts par ces canaux d'informations sont pour l'instant peu étendus et concernent seulement certains aspects de la veille médicale et sanitaire.

4) Le fil RSS : Avec le développement des blogs spécialisés, le RSS (Rich Site Summary ou Really Simple Syndication, selon les traduc- 
tions) permet de recevoir automatiquement un lien généré à chaque mise à jour du site surveillé. Le flux RSS indique ainsi les nouveautés et permet de se tenir informé sans être obligé de visiter régulièrement les mêmes sites. Ces flux RSS peuvent être lus via un navigateur web ou un logiciel de messagerie. Il existe également des « agrégateurs " qui permettent de recevoir, en un seul et unique endroit, tous les fils RSS auxquels l'utilisateur est abonné.

Ces divers outils se différencient entre eux par le type de méthode employée pour la veille. Les spécialistes distinguent deux grands types désignés par leur appellation anglaise : "pull» (tirer) et "push » (pousser).

1) La méthode "pull » consiste à se rendre directement et régulièrement sur les sources d'informations (site Web, blog, portail, etc.) pour en " tirer " les nouvelles les plus récentes dans un domaine particulier. L'utilisateur établit un profil (une référence, un choix de champ d'intérêt, des titres de revues, etc.) pour chaque outil sélectionné (bases de données, moteurs de recherche, éditeurs de périodiques, etc.) et ce profil va lui permettre de constituer un dossier documentaire de façon rapide et ciblée.

La méthode "pull » est généralement utilisée pour les bases de données non structurées ou pour les sources d'information qui n'offrent pas de service de signalement automatique. Le veilleur doit donc relancer la recherche régulièrement, s'il désire rester informé des nouvelles publications dans son domaine.

2) La méthode "push " consiste à apporter l'information à l'utilisateur ou encore à " pousser " vers lui, de manière directe et automatique, des informations qui appartiennent à des sujets préalablement sélectionnés. Ces informations proviennent des bulletins d'informations (newsletters), des listes de diffusion (mailing lists) et autres services d'alertes. Dans la méthode "push", l'utilisateur se contente donc de s'abonner à des services de veille qui font le travail à sa place. 
Ces deux méthodes de veille sont déjà utilisées pour le domaine sanitaire et médical, mais les professionnels de la santé recourent de plus en plus aux agents intelligents parce qu'ils sont plus précis et plus efficaces.

Les agents intelligents sont des robots de recherche, plus ou moins autonomes, qui assistent le spécialiste de la veille dans le suivi de l'information spécialisée. Ils opèrent soit directement en lieu et place du spécialiste, soit indirectement en lui offrant des choix à partir d'une connaissance partielle des objectifs de veille.

Selon la définition de l'AFNOR, un agent intelligent est un « objet utilisant les techniques de l'intelligence artificielle. Il adapte son comportement à son environnement, et en mémorisant ses expériences, se comporte comme un sous-système capable d'apprentissage : il enrichit le système qui l'utilise en ajoutant, au cours du temps, des fonctions automatiques de traitement, de contrôle, de mémorisation ou de transfert d'information".

En règle générale, un agent intelligent contient deux éléments principaux : une base de connaissance prédéfinie et un moteur d'inférence pour générer des raisonnements plus ou moins complexes. Cela lui permet notamment de remplir les fonctions suivantes : la recherche " intelligente " d'informations, l'analyse linguistique des informations récupérées, l'indexation sémantique des résultats (ex. EchoSearch), le résumé automatique des données récupérées (ex. WebCompass), le filtrage et l'archivage de résultats (ex. WebSeeker), etc.

Le grand intérêt des agents intelligents pour la veille sanitaire et médicale réside dans leurs caractéristiques intrinsèques qui ne sont pas disponibles dans la veille " classique " :

1) L'autonomie : L'agent est capable de prendre des initiatives et d'agir sans intervention de l'utilisateur. C'est le cas, par exemple, des logiciels d'Arisem (www.arisem.com), de Wysigot (www.wysigot.com) ou encore de Temis (www.temis-group.com) pour le domaine des sciences de la vie. Le logiciel se connecte au Web, à intervalles régu- 
liers et de façon automatique, pour y effectuer les tâches qui lui sont confiées. Ensuite, il signale à l'utilisateur le résultat de ses recherches et lui présente l'information sous un format ergonomique.

2) La coopération : L'agent intelligent est capable d'échanger des informations avec des serveurs ou avec des humains. Ainsi, un agent représentant un utilisateur peut échanger de façon automatique des informations avec d'autres agents représentant d'autres utilisateurs. Au fur et à mesure que le nombre d'utilisateurs augmente, le nombre des agents et la quantité des informations échangées augmentent en même temps. L'agent coopère ainsi à l'accroissement des connaissances des autres et récupère une quantité d'information de plus en plus grande et spécialisée.

3) Le raisonnement : L'agent est capable de s'adapter à son environnement, qui peut être composé d'autres agents, du web en général ou des utilisateurs humains. Cette adaptation s'appuie sur l'analyse de l'environnement à partir de règles d'inférence qui imitent la logique humaine et le fonctionnement des experts lorsqu' ils recherchent une information nouvelle sur un domaine particulier.

4) La mobilité : Les agents intelligents sont multi-plateformes et multi-architectures, c'est-à-dire qu'ils peuvent fonctionner sur différents systèmes et se déplacer sur le réseau des réseaux pour accomplir les tâches de veille sans que l'utilisateur s'en aperçoive.

Grâce à ces caractéristiques, les agents intelligents permettent d'analyser en profondeur les documents et d'extraire l'information pertinente, de la classer automatiquement et de la structurer rapidement. Certains logiciels utilisent une technologie linguistique fondée sur des classifications sémantiques prédéfinies, mais la plupart sont issus des systèmes experts développés par des médecins ou des professionnels de la santé reconvertis dans l'ingénierie des connaissances.

En raison de leur spécialisation accrue, ces logiciels permettent de générer l'activité de veille en fonction du contenu : institutionnel (médico-administratif), clinique (médico-scientifique), stratégique (médico-économique). 
En pratique, les agents intelligents récupèrent toutes sortes d'informations classées par rubriques : les nouveautés des agences sanitaires, les textes officiels et réglementaires, les recommandations et consensus, l'actualité des praticiens de santé, les alertes sanitaires, les articles scientifiques, les essais cliniques, les articles de la presse quotidienne nationale, les sommaires de la presse médicale internationale, etc.

Voici, à titre d'exemple, deux sites Web de veille qui utilisent la technologie des agents intelligents et qui montrent les possibilités offertes aujourd'hui à tous ceux qui souhaitent s'engager dans ce domaine innovant (la veille) et se spécialiser dans un secteur d'activité en pleine expansion (la veille sanitaire et médicale).

1) Webvigie (www.webvigie.com) est un logiciel gratuit qui surveille le web et avertit l'utilisateur par mail lorsque le contenu d'un site a changé. En pratique, on entre l'adresse du site à surveiller et l'on précise la fréquence de passage de Webvigie sur la page. Le site intègre un agent de recherche intelligent qui a été développé par un étudiant en médecine. Son intérêt réside dans le fait qu'il n'a pas de limite dans le temps, c'est-à-dire qu'il va chercher même les pages effacées ou supprimées. Webvigie envoie un mail à chaque fois que le site surveillé change de contenu.

2) Pertinence (www.pertinence.net) est un outil d'analyse linguistique qui produit des résumés automatiques de textes sur Internet. Il suffit pour cela d'indiquer l'adresse Internet (URL) du texte à résumer. Son utilité tient au fait qu'il permet de résumer automatiquement un texte tout en tenant compte de son domaine thématique. Une liste des termes est présentée avec le résumé. Les domaines pris en compte par le logiciel sont : la médecine, mais aussi la chimie et la finance. Les langues traitées pour ces domaines sont : le français, l'anglais, l'allemand, l'espagnol, l'italien, le portugais. Ce site montre bien les potentialités de la veille multilingue en matière de santé publique. 


\section{Conclusion}

La veille sanitaire et médicale a pour but de faciliter l'identification des problèmes pertinents et de maintenir un flux régulier d'informations appropriées dans le domaine de la santé publique et de la gestion hospitalière. Les outils présentés ici procurent un gain de temps considérable et une visibilité réelle concernant les données stratégiques. Ils permettent de traiter des milliers de documents et de personnaliser l'activité de veille face au flot incessant d'informations. Bref, grâce à ces outils, le travail de veille devient à la portée de tous.

Face à l'évolution constante des technologies et des sources d'information, cette veille devra s'adapter aux besoins des médecins et des professionnels de la santé. Actuellement, la veille la plus efficace est offerte en anglais, plus précisément en anglo-américain, mais les systèmes de veille francophones ouvrent activement pour rattraper leur retard. Sans aucun doute, la veille de demain sera multilingue et multifonctionnelle.

\section{Bibliographie}

Hauray, B., 2006, L'Europe du médicament. Expertise - politique - intérêts privés, Presses de Sciences Po, Paris.

Numéro spécial, 1999, "Les pouvoirs publics et la sécurité sanitaire ", Revue française des affaires sociales (1).

Numéro spécial, 1999, "Sécurité et risques alimentaires ", Problèmes politiques et sociaux (823) : 3-75.

Abenhaim, L., 2003, Canicules. La santé publique en question, Paris, Fayard.

Capp, G., 2001, La sécurité sanitaire des aliments : un enjeu majeur, Paris, Conseil économique et social.

Delomenie P. and A. Flahaut, 1997, "Les vigilances sanitaires ", Revue française des affaires sociales 12 (3-4) : 91-106. 
Lalande, F., 1997, "Sécurité et veille sanitaires : concepts, historique, évolution des structures et des doctrines ", Revue française des affaires sociales (3-4) : 31-38.

Roqueplo, P., 1996, Entre savoir et décision, l'expertise scientifique, Paris, INRA-éditions.

Tabuteau, D., 2002, La sécurité sanitaire, Paris, Berger Levrault. $2 \mathrm{e}$ édition. 